\title{
Mixed Reality , Applications in Neurodiversity.
}

Dr Bheemaiah, Anil Kumar, A.B Seattle W.A 98125

miyawaki@yopmail.com

\section{Abstract:}

Technology has rendered many of the accommodations needed for ASD, possible with a recognition of Neurodiverse individuals as a minority, with a cultural context of their own. Technology renders special education obsolete, while with the accommodation technology provides, the autistic minority, the neurodiverse can lead a lifestyle no different from the neurotypical. Mixed Reality is one of those technological marvels, with wearables, creating mixed reality, both real and augmented, adding an artificial synthesia to a natural one. In this paper we consider 19 such papers, with 5 reviews proving the efficacy of mixed reality and the rest in technology of mixed reality and applications in neurodiversity accommodations and education.

Keywords: neurodiversity, mixed reality, Redmond, workforce, PD, participatory design

\section{Introduction.}

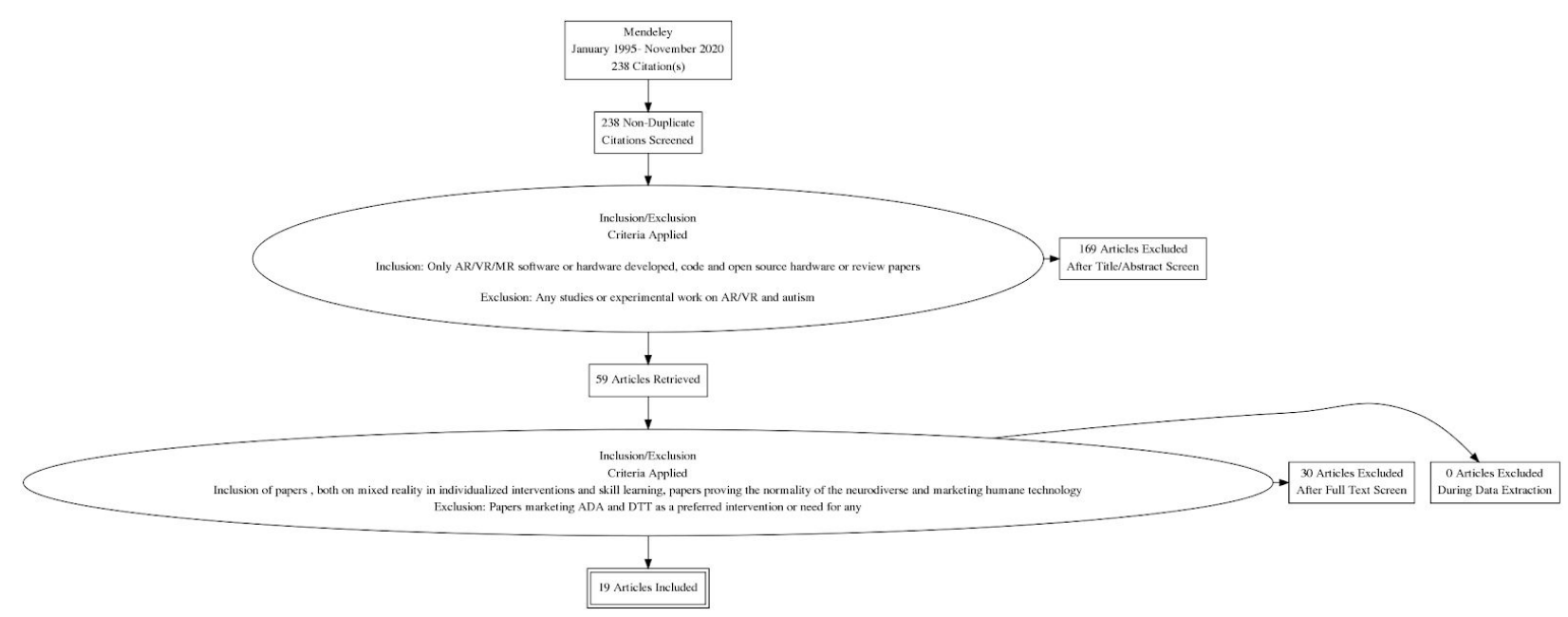

Fig 1: PRISMA diagram for Systematic review for MR in symmetric education for neurodiverse and neurotypical with technological accommodations on demand. 


\section{Rationale:}

The digital divide exists across society, towards a more secular digitally literate society, where technology has rendered the need for interventions for the neurodiverse obsolete, with digital literacy comes a spectrum of technology and on demand accommodation engineering, allowing the neurodiverse to live and perform within the neurotypical community and accepted as a normal minority culture. In this paper we explore published literature towards digital literacy, and evidence of the technological obsolescence of the need for interventions, transitioning or individualized special education.

\section{Objectives.}

The research problem is centered on the hypothesis $\mathrm{HO}$ :

$\mathrm{HO}$ : Mixed Reality is effective in creating sufficient accommodation engineering, rendering a transition program obsolete, allowing for neurodiverse individuals to live in the mainstream world of neurotypicals.

In the PICO formulation, in the context of the neurodiverse population is the obsolescence of interventional strategies with positive outcomes of the need and a strategic plan at digital literacy of both neurotypical and neurodiverse populations, identification of markets for specialized and social technology and a gradual addition of these accommodations on demand in all digital technology.

\section{Methods.}

Eligibility criteria: Technology centric publications and review papers on mixed reality and autism, from January 1995 to November 2020 were considered.
Information sources: Several databases included ACM, IEEE Spectrum, Mendeley, Google Scholar and Paperpile.

\section{Search strategy:}

Mendeley, a cumulative search tool was used, exporting the references to paperpile for abstract and full text based scanning, for inclusion, preferred to tools like abstract. Several data ming and manual data extraction methods were compared.

Data management: Paperpile provides a comprehensive data management tool, supplementing mendeley. The libraries allow for selective persistence of search results and citations to an automated bibliography, imported to paperpile from mendeley.

\section{Selection process:}

Abstract and full text scans for reviews and pure technological content, minimizing experimental findings. All texts can be indexed and browsed for pre screening on paperpile.

\section{Data collection process}

Use of extended grep and text rank based tools for data selection, text extraction, summarization and search based on five or more keywords. Summarizers extend the basic unix grep like functionality to more than one keyword search and summarization of the search results. This text mined data is used to manually compose a review.

\section{Outcomes and prioritization}

Outcomes are non interventional, proxemic, open ended, game - interactive and social. In an attempt at academic distancing from the outdated deficit based interventional approach to a neurodiversity approach, neurodiversity friendly outcomes are selected from the search.

\section{Risk of bias in individual studies}

Since experimental studies on mixed reality technologies were excluded, experimental bias is irrelevant to the present publication.

Since clinical studies are excluded in this review, an analysis of evidence for experimental bias, and meta regression, sensitivity and grouping analysis are not 
included. Publication bias and selection bias are also not included. A mere coverage test is included for a selection filter to span the entire skill set in transition definitions for neurodiverse populations proving that for any such skill, accommodation on demand is simply the issue of digital literacy.

\section{Background.}

[15] describes technological support for the neurodiverse, lack of eye contact amongst neurodiverse individuals is addressed with cartoon animated faces, kintoon using projected cartoonizer based avatars with artificial makeup, this makeup is proven to bridge the gap between the neurotypical and neurodiverse, eliminating the inability to maintain eye contact.

Competing are display based cartoonish faces like the Moxie,[16] social robots for neurodiverse individuals and animated soft toys called Cuddles[4,5], developed by the author.

Classically, ABA Therapy with DTT, for task learning with Trails was adopted and mixed reality used to create an impression in the ABA formalism, as described in[2]formalism and automation in a reasoning ontological system for automation of $A B A$ using social robotics, the author differs, in humane technology for the neurodiverse.[17]

An approach of fully immersive VR with cave displays is described in [6]

It inculcates open ended game playing, face to face interactions and proxemics, the need to negotiate subconsciously for one's space, a skill every neurodiverse needs in interacting with the mainstream neurotypical.

[1] use social stories as skills with VR based scenarios in community settings for social skills, proving community activities, can be in mixed or virtual reality as a precursor to exposing neurotypical or neurodiverse individuals to the marketing based society, from protected circles.

[14] use a visual aid for flash card based pedagogy for interventional learning of chinese words for communication skill development.
[10] prove the viability and usability of multimodal augmentation and virtual reality with sensor fusion in special education and social networking in the neurodiverse population. Using gaze tracking and PPG data, an individualised Intervention with social scenarios is developed as a paradigm.

\section{Systematic Reviews:}

[9] presents a systematic review, with 30 publications on AR/VR or MR interventions on learning skills in neurodiverse populations ranging from social skills to day to day living skills with inconclusive evidence to prove the effectiveness of AR or MR, calling for further clinical research. [8] in a review in technology for the neurodiverse, cites a definite advantage in the use of technology ranging from VR and MR to social robotics, proving that it gives the neurodiverse an opportunity to express themselves, and be accepted as themselves a cultural minority, with no requirements for a cure or intervention.

\section{MR and NeuroDiverse.}

In [13], the place of advanced technology, both in education and at the workplace are described, citing recent progress as Redmond as an example of the increased acceptance of the strengths of the neurodiverse in corporations across the globe, added to this are several studies of successful education of practical life skills from using a airport, calling a shared ride, using public transit, crossing a street and many essential life skills taught through VR or MR based education.

[11] is a technological paper on design and usability of $U I$ and $U X$, to include the neurodiverse, in a strong statement on the acceptance of the neurodiverse as they are, as part of an ecosystem, the author argues for an extra mile in technology design to incorporate usability information by the neurodiverse community. Open ended games are used to teach optional skills like self-regulation, attention, and communication skills.

[7], describes controversies in design centered around the neurodiverse, agnostic PD or participatory design is centered around two groups of hyper-creative neurodiverse children for the design of social play MR technology, the author attempts to distance himself 
from reconciling opposite viewpoints in play, motivations and individualization and creates a new paradigm in design with controversies.

[3] introduced participatory design and D4D, diversity for design, a process of involving the participants in the design process, in two case studies, the authors contribute invaluable PD design thinking, especially useful to design for both the neurotypical and neurodiverse. A application called TEACCH is developed by the neurodiverse for the neurodiverse population.

[12] is a strong advocacy for the neurodiversity movement citing the Auton Vs British Columbia case to an end to stereotypes of neurodiverse as a disorder, needing remediation, an end to an abduction of innocence, and a transition to a postmodern society of larger creative ecosystems, with an acceptance of a neurodiversity and of technology.

The research indicates a trend for a dominance of the adaptation of a strength centered transition for the neurodiversity population and increased participation in newer design paradigms, for this user group. With time, and better design information for this user group, nearly all digital technology will have a high usability by the neurodiverse.

\section{Results.}

Given a linear scan on a list of skill categories, (communication, life, social, financial, mobility, technical, knowledge, creative arts) we have found evidence that technology can accommodate neurodiverse populations in all of these skills with a strength based IEP, both in a curricular and an informal context. The state of art technologies in digital literacy include $\mathrm{BCl}$, voice based $\mathrm{UI}$ and several UI technologies with mixed reality using cave systems, SAR systems and head mounted displays. The hypothesis is proven with future trends in technology predicted when all digital technology will incorporate the accommodations needed for the neurodiverse and pervasive A.I already pervasive today will be applicable to all the markets where neurodiverse customers exist.

\section{Discussion.}

A review of technology of mixed reality applications in autism interventions are presented, with systematic reviews, with mixed verdicts on the usefulness of mixed reality interventions for skill acquisition in neurodiverse individuals. The author predicts the natural incorporation of the needed accommodation into all technological products, not necessitating the need for intervention or transitioning.

\section{Future Work.}

A live review of accomodation on demand in operating systems, smart home technologies and other digital technologies through humanising technologies like Amazon Pollexy, towards a social robotics and mixed reality approach, away from the ADA and DTT formulation is described. This publication thus reviews several publications supporting the primary hypothesis and proving the hypothesis of participatory design and advancement of technology in assisting neurodiverse populations, the minority culture of neurodiversity is successfully empowered with marketing of their strengths , a movement so successful, indicated by workshops at Redmon and several other corporations on hiring and keeping a neurodiverse workforce.

\section{References.}

[1] Ali Adjorlu, Aishah Hussain, Camilla Mødekjær, and N. Warming Austad. 2018. Head-mounted display-based virtual reality social story as a tool to teach social skills to children diagnosed with autism spectrum disorder. In 2017 IEEE Virtual Reality Workshop on K-12 Embodied Learning through Virtual \& Augmented Reality (KELVAR). IEEE. Retrieved from https://pdfs.semanticscholar.org/e752/68d7a1dfb316996 a78233678a7eb5c0eec68.pdf

[2] Edmon Begoli. 2014. Procedural Reasoning System (PRS) architecture for agent-mediated behavioral interventions. In Conference Proceedings - IEEE SOUTHEASTCON.

DOI:https://doi.org/10.1109/SECON.2014.6950715

[3] Laura Benton, Asimina Vasalou, Rilla Khaled, Hilary Johnson, and Daniel Gooch. 2014. Diversity for design: a framework for involving neurodiverse children in the technology design process. In Proceedings of the SIGCHI Conference on Human Factors in Computing Systems (CHI '14), Association for Computing Machinery, New York, NY, USA, 3747-3756. DOI:https://doi.org/10.1145/2556288.2557244 
[4] Anil Kumar Bheemaiah. Engineering Psychology, Taskoids for Cuddlies(TM), Role in Autism Therapy. DOI:https://doi.org/10.31234/osf.io/hpjx5

[5] Anil Kumar Bheemaiah. Plants and IOT, PEMF Qi generation as Plant Energetics, an Autism case study. DOI:https://doi.org/10.31234/osf.io/t6sjd

[6] Ciera Crowell. 2018. Analysis of Interaction Design and Evaluation Methods in Full-Body Interaction for Special Needs. In 23rd International Conference on Intelligent User Interfaces (IUI '18), Association for Computing Machinery, New York, NY, USA, 673-674. DOI:https://doi.org/10.1145/3172944.3173150

[7] Christopher Frauenberger, Katta Spiel, Laura Scheepmaker, and Irene Posch. 2019. Nurturing Constructive Disagreement - Agonistic Design with Neurodiverse Children. In Proceedings of the 2019 CHI Conference on Human Factors in Computing Systems (CHI '19), Association for Computing Machinery, New York, NY, USA, 1-11. DOI:https://doi.org/10.1145/3290605.3300501

[8] Naren J, Vithya G, Srivathsan S, and Bharath Reddy. 2019. Supporting Individuals with Autistic Spectrum Disorder with Various Technologies: A Review. International Journal of Psychosocial Rehabilitation 23, 1 (2019). DOI:https://doi.org/10.37200/ijpr/v23i1/pr190242

[9] K. Khowaja, B. Banire, D. Al-Thani, M. T. Sqalli, A. Aqle, A. Shah, and S. S. Salim. 2020. Augmented Reality for Learning of Children and Adolescents With Autism Spectrum Disorder (ASD): A Systematic Review. IEEE Access 8, (2020), 78779-78807. DOI:https://doi.org/10.1109/ACCESS.2020.2986608

[10] Stephanie Lackey and Randall Shumaker. Virtual, Augmented and Mixed Reality. Designing and Developing Virtual and Augmented Environments. In Virtual, Augmented and Mixed Reality. Designing and Developing Virtual and Augmented Environments (Lecture Notes in Computer Science), Springer International Publishing, Cham. DOI:https://doi.org/10.1007/978-3-319-07458-0

[11] Vivian Genaro Motti. 2019. Designing emerging technologies for and with neurodiverse users. In Proceedings of the 37th ACM International Conference on the Design of Communication (SIGDOC '19), Association for Computing Machinery, New York, NY, USA, 1-10. DOI:https://doi.org/10.1145/3328020.3353946

[12] Michael Orsini. 2012. Autism, Neurodiversity and the Welfare State: The Challenges of Accommodating Neurological Difference. Can. J. Polit. Sci. 45, 4 (2012), 805-827. Retrieved from http://www.jstor.org/stable/23391057

[13] Brenda K. Wiederhold. 2020. Our Neurodiverse Society: The Role of Advanced Technology. Cyberpsychol. Behav. Soc. Netw. 23, 1 (January 2020), 1-2. DOI:https://doi.org/10.1089/cyber.2019.29171.bkw

[14] Pinata Winoto. 2016. Reflections on the Adoption of Virtual Reality-based Application on Word Recognition for Chinese Children with Autism. In Proceedings of the The 15th International Conference on Interaction Design and Children (IDC '16), Association for Computing Machinery, New York, NY, USA, 589-594. DOI:https://doi.org/10.1145/2930674.2936001

[15] Cheng Zheng, Caowei Zhang, Xuan Li, Fan Zhang, Bing $\mathrm{Li}$, Chuqi Tang, Cheng Yao, Ting Zhang, and Fangtian Ying. 2017. KinToon: A Kinect facial projector for communication enhancement for ASD children. In UIST 2017 Adjunct - Adjunct Publication of the 30th Annual ACM Symposium on User Interface Software and Technology. DOI:https://doi.org/10.1145/3131785.3131813

[16] Full Page Reload. Retrieved November 27, 2020 from https://spectrum.ieee.org/automaton/robotics/home-robo ts/moxie-a-social-robot-for-childhood-development

[17] For Technologists. Retrieved November 27, 2020 from https://www.humanetech.com/technologists 